\title{
Intelligence Cultivation \& Contextual Analysis \& Literature Appreciation On the Instructing and Applied Value to Chinese Teaching of Linguistic Theories
}

\author{
Jiugen $\mathrm{Xiao}^{1, \mathrm{a}^{*}}$,Ying Zhou ${ }^{2, \mathrm{~b}}$, Guangyu $\mathrm{Yu}^{3, \mathrm{c}}$
}

Jiangxi Normal University Language and Language Life Research Center, Nanchang, Jiangxi, China

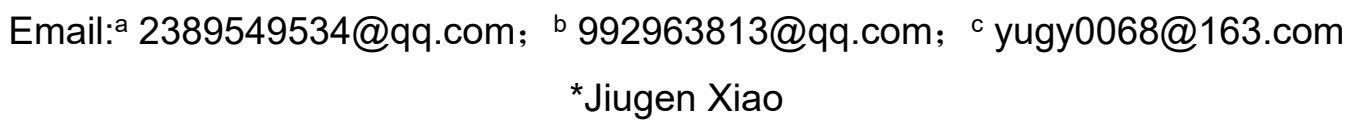

Key words: Linguistic theory ; Chinese teaching ; Instruction; Applied value

\begin{abstract}
Basic linguistic theory comes from practice, and in turn it serves practice.It is very important as well as many other subjects, from the intelligence training of language teaching and context analysis to the literature appreciation.None of them can separate from it. Besides, there also exist many practical application. Among all of these, just like a key to the door, the instruction of linguistic throry is of great importance and application value to Chinese teaching practice.
\end{abstract}

\section{智能培养 $\cdot$ 语境分析 $\cdot$ 文学欣赏 一论语言学理论对语文教学的指导作用及应用价值}

肖九根 $1, \mathrm{a}^{*}$ ，周颖2,b，余光显 $3, \mathrm{c}$

江西师范大学 语言与语言生活研究中心, 南昌, 江西, 中国

Email: 2389549534@qq.com, b992963813@qq.com, cyugy0068@163.com.

*肖九根

关键词：语言学理论; 语文教学; 指导作用; 应用价值

提 要: 语言学基本理论来源于实践, 又服务于实践, 它与多种学科都有着密切的关系, 其 实际应用也是多方面的。在诸多关系之中, 语言学理论对语文教学实践显得尤为重要, 从语 文教学中的智能培养、语境分析到文学欣赏, 无论那一方面都离不开这一理论, 它就像一把 打开语文教学大门的钥匙, 具有重要的指导作用和应用价值。

\section{1. 引言}

语言学是研究语言现象和语言规律的一门科学。这一科学理论来源于实践, 又服务于实 践。在人们的实践活动中, 语言学理论的指导作用是多方面的, 其应用领域也是广泛的, 它 不仅与哲学、社会学、人类学、历史学等社会科学关系密切, 还同生理学、物理学、心理学、 神经学等许多自然科学也有联系。在诸多的关系之中, 我们认为它对语文教学实践尤为重要, 是语文教学中的理论武器, 具有重要的指导意义和应用价值。

语言学理论与语文教学关系究竟如何, 它在语文教学中具有怎样的指导意义和应用价值, 我们择其二三，谈一点粗浅的认识。 


\section{2. 语言学理论在语文教学中对学生的智能培养具有十分重要的作用}

语言学基本原理告诉我们, 语言是音义的结合体, 是人们意识活动的物质手段, 它有助 于人们认识物质世界, 在人们的认识过程中起着十分重要的作用。众所周知, 人对客观世界 的认识即反映, 是一个由低级向高级不断深化的过程。这个过程要经过感觉、知觉、表象和 思维几个基本环节, 这也是人们对事物的认识由感性上升到理性的必经阶段。尽管各阶段的 作用不同, 但作为认识事物的基本形式必须在语言的参与下才能实现它的反映, 不论是直接 的还是间接的, 也不论是具体的还是抽象的。思维是认识过程中的一个核心, 它能使人们对 事物的认识由感性上升到本质认识。语言对思维这一认识形式在反映事物的过程中显得特别 重要, 除其基本过程需要语言参与外, 它的基本形式一一概念、判断、推理的形成与实现, 也离不开语言。

语言在认识过程中不仅作用于抽象思维, 还作用于形象思维, 这是同一事物的两个方面。 形象思维不直接运用概念, 而是运用感知经验和表象活动来反映外在事物的联系与规律, 是 一种在理性认识控制下的基本形式。由于人的理性认识是借助语言符号而存在的, 所以理性 认识对形象思维的控制具体表现为语言系统的控制。换句话说, 理性认识对形象思维的控制 是以语言为其物质手段的。事实上, 在人们反映事物的过程中, 抽象的“理性思维”要受到意 识的感性环节支持、作用, “理性思维”从来就不是超感知、超表象的; 另一方面, 运用感知 经验、表象活动的形象思维, 也不是与抽象的理性认识无关, 而是在理性认识控制之下进行 的。语言对这两种认识形式是不可缺少的, 只是所起的具体作用不同而已。在抽象思维中, 语言是作为直接的操作手段; 在形象思维中, 语言则是一种控制手段。没有这种物质手段的 作用，人们就不可能完成对事物的反映。斯大林曾经说过: “不论人底头脑中会产生什么样的 思想, 以及这些思想在什么时候产生, 它们只有在语言的材料底基础上、在语言的术语和句 底基础上才能产生和存在。”[1]

语言是思维的物质外壳, 而思维又是智能的核心因素, 这三者共处于一个统一体。所谓 智能, 就是以思维为核心的各种能力的综合 (包括观察力、注意力、思维力、想象力、记忆 力等等), 实质上是大脑对信息加工的能力。根据语言学理论, 语言教育涉及到对青少年思 维的优化培养与训练, 这是发展他们智能最重要、最基本的手段。如果他们没有受到良好的 语言教育, 其思维能力就得不到充分发展, 大脑的信息加工能力就要受到影响, 这就意味着 智能发展受到限制。语言是思维的工具, 是意识存在与意识活动的物质手段。没有语言, 就 没有思维活动, 也没有意识和意识的整体活动, 感性认识也不会上升为理性反映形式的认知。 也就是说, 大脑对信息的加工、对事物的反映就不可能得以实现, 其原因是大脑对信息的加 工, 必须借助语言符号的作用。离开了语言符号, 这种信息加工是不可能的, 人类也就不可 能有自己特有的智能活动, 不可能取得对外部事物的认知。对于个人而言, 语言越是得到发 展, 他对事物或现象的分析、综合能力就越强, 他的智能就越能得到发展。可以这样说, 语 言是人的智能活动必不可少的要素, 是人们摄取外部信息进行加工不可或缺的手段。的确, 人的智能水平是由多种因素决定的, 但语言发展水平对促进智能的发展却是一个十分重要的 因素。

语文教学是对青少年进行语言教育的一种实践活动, 也是培养青少年智能的一项最基本、 最重要的活动。这一活动是由语文科的性质、体系、内容所决定的。“语文”是一种语言科学, 即口头语言和书面语言的结合体, 它的知识结构是以语言学、文章学、文学、逻辑学等学科 的理论为基础, 其内容包括字、词、句这些基本语言材料和听说读写知识、文章知识以及语 音、文字、词汇、语法、修辞诸方面的知识。语文科的教学对象、任务、规律也决定了语文 科的教学特点。如中学, 一般是十二、三至十八、九岁的青少年, 他们思维敏捷, 反应快, 正处于发展变化时期, 可塑性强, 对他们进行语言教育, 能使他们受到良好的思想陶冶, 提 高他们对美好事物的感受能力, 更为重要的是培养他们听说读写能力, 促使他们思维的快速 发展, 从而提高他们的智能水平。这是符合实现语文教学科学化、高效化基本原则的, 也是 
进行语言教学最终要达到的目标。语文教学是以语言活动为基础, 而语言活动又会大大促进 思维的发展。这是语文教学中听说读写离不开感知过程, 离不开理解过程, 也离不开记忆过 程的缘故; 而整个过程中, 包括表达过程都始终贯穿、伴随着思维活动。不可置疑, 语文活 动是一种以智力为前提条件、以思维为核心的智能活动。另一方面, 语言是产生和发展人类 思维的主要动力。掌握语言, 发展语文能力, 不仅是学生获得知识经验的必要条件, 也是他 们思维尤其是智能发展的重要条件。由于语言的构成及其能力的形成, 是世代系列社会进步 信息与优化思维的集合, 因而语文教学包含着对受教育者摄取进步信息能力以及优化思维能 力的培养, 是形成青少年智能的十分重要的因素。在语文教学中, 如果一个教师有意识地把 语言训练和思维培养紧密地结合起来，这对增进青少年的智能是大有裨益的。

\section{3. 语言学理论在语文教学中对学生作具体语境下的语义分析具有十分重要的作用}

语境是语言学理论中的又一基本问题, 它在语言教学中起着一定的制约作用, 能“使语义 图式中不充分赋值的语义关系获得具体的意义解释”。[2]语言教学是以分析语言的基本材料和 基础知识为主要内容的, 而语言又是音义构成体。语音是语言的形式, 语义是语言的内容。 内容决定形式, 反映事物的本质。因此, 我们分析语言, 应重视语义的分析和研究, 尤应重 视具体语境作用下的语义分析研究。

语境谓之语言环境, 既包括特定的言谈现场、话语语境, 也包括特定的社会文化背景知 识等。[3]“就语言发生学而言, 任何语言现象都出现在特定的语境当中, 并且通过组合、聚合 等线性或非线性手段最终成为表达特定意义的符号形式。”[4]在实际的语言教学中，我们既要 分析语言中的静态语义, 更要分析具体语境中的动态语义。也就是说, 我们要把语言体系本 身所具有的词汇意义和语法意义同语言单位在特定语言环境中实际所传递的信息结合起来分 析。而语境是句子动态语义的基础, 人类语言行为的多变性决定了语境的多样性, 语境的多 样性又决定了它对生成语言单位动态语义作用的多方面。关于语境对语言的影响和作用, 人 们早就注意到了。20世纪60年代, 转换生成语言学大师乔姆斯基在他的转换生成理论中就提 出了“语境自由”和“语境制约”的理论。

那么, 语境对于理解句子的动态语义究竟有何意义呢? 下面我们主要以语文课本为例来 简要分析这一问题。

首先，利用语境有助于推断句子的言外之意。例如:

老梁歪着脑袋看着儿子, 怎么这么不顺眼? 啊, 对了! 黑眼镜！他呼地伸出手, 扯下儿 子的眼镜, 啪地摔到地上: “这玩艺儿叫你看不清! ”

“看不清”字面上是指带墨镜看不清, 骨子里是说有了坏思想就会看不清前进的方向, 就 会走上邪路。

鲁迅在《阿长与山海经》中有一段话:

一到夏天，睡觉时她又伸开两脚两手，在床中间摆成一个“大”字，挤得我没有余地翻身， 久睡在一角的席子上, 又烤得那么热。推她呢, 不动。叫她呢, 又不闻。

“长妈妈，生得那么胖，一定很怕热罢？晚上的睡相，怕不见得很好罢！ .”亲听到 我多次诉苦之后, 曾经这样问她。我也知道这意思是要她多给我一些空席。

“我”的母亲想指出阿长挤得“我”没有地方睡觉，要她多给“我”一些空席，又不好直说， 只好拐弯摸角地说“晚上的睡相，怕不见得很好罢”。

又如, 鲁迅《故乡》里有这样的句子:

我似乎打了一个寒噤; 我就知道, 我们之间已经隔了一层可悲的厚障壁了......我只觉得 我四面有看不见的高墙......

这些语句若不置于具体的语境里, 就不会有这样的理解: 封建社会等级制度和尊卑观念 的毒化, 造成了人与人之间的冷漠, 相互不信任。“这正如地上的路; 其实地上本没有路, 走 
的人多了, 也便成了路”, 也不会理解为: 地上的路, 其实是指新的生活道路, 人生的希望之 路。

其次, 利用语境有助于推断与句子意义相反的信息。例如:

《黛玉之死》写宝玉成亲那一天, 林黛玉气息奄奄, 晚上出现回光返照现象。这时, 林 黛玉紧紧拿住紫娟的手, 使劲地说:

我是不中用的人了！你伏侍我几年，我原指望咱们两个总在一起，不想我......

极度的悲伤和虚弱使她说不下去了。过了好一会, 她才直声喊叫起来:

宝玉！宝玉！你好......

说到“好”字两眼一翻就死了。弥留之间, 一时找不到恰当的言辞, 满腔怨愤绝望凝聚 成“你好......”这两个字。这个“你好......”的后面隐含着痛骂宝玉负心的话语, 被后人叹 为神妙之笔。

又如，鲁迅在《记念刘和珍君》里写道:

中国军人的屠戮妇贞的伟绩, 八国联军的惩创学生的武功, 不幸全被这几缕血痕抹 杀了。

从上下文来看，作者在这并非赞美那些刽子手，而是揭露批判他们滥杀无喜的残暴行径。 这样的例子很多, 兹不一一列举。

再次，利用语境还能推断语句中的准确信息。例如:

曹禺《日出》里有这样的对话:

陈白露 这个东西, 简直——好, 你去吧。

王福升 是。

旅馆的茶房王福升跑来告诉陈白露, 说张乔治醉酒吐了她一床。陈白露听了极为恼火, 正想发作, 猛然想起王福升也不是个好东西, 所以一说出“这个东西, 简直—— ”时, 就赶紧 把话打住。对方当然听得出, 下边没说出来的话是臭骂纳绔子弟张乔治的, 只是没说出来, 别人抓不到把柄而已。

在《生命的支柱——张海迪之歌》中, 作者在叙述了张海迪战胜消极悲观思想之后, 满 怀激情地写道:

对啊, 不排除杂质, 怎么能炼出纯钢呢!

文中的“杂质”是指各种消极悲观的思想情绪, “纯钢”则指坚强不屈的战士, 生活的强者。 没有具体语境的制约，也就失去这种语意了。

很显然, 语境在句子的语义分析中是举足轻重的, 要透彻地理解一个句子的实际意义, 绝不可擞开具体的语境。否则，那将是徒劳的。

著名语言学家张志公先生说: “语言总是在一定的交际环境中使用的, 因此, 分析语言现 象必须把它和它所依赖的语境联系起来, 离开一定的语境, 把一个语言片断孤立起来分析, 就难于确立这个语言片断的结构和意义。"[5]这就精辟地阐明了分析语言现象与语境的辩证关 系。有的人甚至认为, 语言交际时所依赖的语境, 就象人呼吸时依赖的空气一样重要。[6]语 言学原理再次告诉我们: 语言中的静态语义 (句子本身的意义) 与动态语义 (句子在特定语 境中实际传递的信息) 常常不完全相同, 甚至是完全不同的。由此看来, 在语言教学中, 我 们不只要注意静态语义的分析, 更要重视动态语义的把握。只有这样, 我们才能真正理解语 句中的真实内涵。

\section{4. 语言学理论在语文教学中对提高学生的文学欣赏水平具有十分重要的作用}

语言学理论除上述作用之外, 还对提高学生的文学欣赏水平也具有十分重要的作用。大 家知道, 文学作品是作家运用语言材料, 通过想象 (包括虚构) 来塑造形象、反映生活、表 达感情的语言艺术。因此, 可以毫不夸张地说语言是文学的第一要素。语言学基本原理告诉 我们, 语言不单是抽象思维的操作工具, 还是形象思维的控制手段。作家在文学创作中是离 
不开这两种思维形式的, 只是形象思维占首要地位而已。我国古代著名文艺理论家刘劦思认为: “文之思也, 其神远矣。故寂然凝虑, 思接千载; 悄焉动容, 视通万里。吟咏之间, 吐纳珠玉 之声; 眉睫之前, 卷舒风云之色: 其思理之致乎! "[7]他认为作家进行文学创作, 其思维活动 是离不开事物形象的。毛泽东同志也说过: “诗要用形象思维, 不能如散文那样直说, 所以比、 兴两法是不能不用的。”[8]事实也是如此, 这正如高尔基所指出的: “想象在其本质上也是对 于世界的思维, 但它主要是用形象来思维, 是“艺术的”思维。”[9]诚然, 文学创作虽以形象思 维为特点, 但这不等于没有抽象思维参与, 事实上两者是相互渗透、相互作用的。戏剧大师 曹禺在谈《日出》的创作时说，在生活中“我看见多少梦愿一般的可怖的人事，这些印象我至 死也不会忘却，它们化成多少严重的问题，死命地突击着我。”“我想用片段的方法写起《日 出》, 用多少人生的零碎来阐明一个观念。”这个观念就是“人之道损不足以奉有余。”[10]而这 观念“人之道损不足以奉有余”就是抽象思维的表现。这就是说, 在创作过程的形象思维中有 抽象思维在起作用。只要深入研究语言现象, 充分认识语言特点, 我们就能更好地理解、欣 赏文学这一语言艺术。

文学这一语言艺术有其特殊性, 它能净化人的灵魂。学生阅读文学作品, 不光能认识客 观事物, 还能受到思想熏陶和审美教育。众所周知, 文学是社会生活的反映, 不论直接的还 是间接的, 都是对现实生活的艺术创造, 而不是简单的复制, 它比普通的实际生活更典型、 更理想、更具普遍性, 因而也就更富有教育意义。文学是以语言塑造形象来反映社会生活的, 而其形象是具体可感的。在具体可感的形象中, 倾注了作家的思想情感。优秀的文学作品是 一部“生活的教科书” (车尔尼雪夫斯基语)，它总是通过生动精粹的语言来创造典型形象。 通过阅读、欣赏这些文学作品, 学生就可以从对社会生活的赞美中、从对丑恶现象的鞭挞中 来认识艺术形象所反映的社会生活的面貌和本质。这样, 他们不只得到了艺术上的享受, 还 能受到思想上的启迪和教育。譬如, 阅读契诃夫的《变色龙》, 学生对那个狡诈多变, 媚上 欺下的沙皇警官奥楚荗洛夫种种丑态, 会虽之以鼻, 并在嘲笑声中激起他们对那沙皇走狗厌 恶、憎恨的情绪。莎士比亚的《威尼斯商人》以揶揄调㑆的笔调, 深刻地揭露了夏洛克贪婪、 吝啬、残忍、固执、冷酷的卑劣行径, 学生读后表现出一种怒不可遏的愤激之情, 认清了夏 洛克极为阴险、十分凶残的丑恶本质。可见, 文学中的反面人物、丑恶事物, 由于作者以正 确观点真实地描写了他们的面貌, 揭露了他们的本质, 这对学生是会起到很好的教育作用。 而杨振宁的《邓稼先》通过回忆两弹元勋邓稼先不平凡的人生经历以及他研制两弹的巨大贡 献, 表现了邓稼先的爱国情怀, 学生被他那英勇无畏、身先士卒的人格品质所感动, 所鼓舞, 所激励。成功的文学作品, 往往是一幅自然景物、社会生活的绚丽画图, 具有很高的艺术审 美价值。作品中的正面人物特别是英雄人物的英勇行为, 能激发学生的思想情感, 使学生感 受到英雄人物的品质美。如《钢铁是怎样炼成的》中的保尔·柯察金，《刑场上的婚礼》中的 周文雍、陈铁军, 《谁是最可爱的人》中的志愿军战士, 早已成为千百万青少年心目中的楷 模, 具有巨大的教育意义和鼓舞力量。列宁称文学的这种作用是一种“教导人、引导人、鼓舞 人”的作用，它能使人们“自觉勇猛发扬精进”（鲁迅语）。高尔基在谈到文学对人们的作用时 也说: “文学的目的就是帮助人了解他自己; 就是提高人的信心, 激发他追求真理的要求; 就 是和人们中间的鄙俗作斗争, 并善于在人们中间找到好的东西; 就是在人们的心灵中唤起羞 耻、愤怒和英勇, 并想尽办法变得高尚有力, 使他们能够以神圣尚美的精神鼓舞自己的生 活。”[11]不过, 无论文学作品有着怎样的特殊功能, 它都离不开语言这个第一要素在其中所起 的作用。懂得了这一点, 我们就会正确地对待文学作品的教学, 就会自觉地把文学作品的教 学看作是语文教学中的一个重要组成部分, 它不但有利于学生扩大知识领域, 提高思想觉悟, 培养审美能力, 更为重要的它还是学生学习语言, 提高语言运用能力的有效途径之一。了解 了这一点, 就不会把语文教学中的“文学因素”和“语言因素”对立起来。这样, 在进行文学作 品的教学中, 我们就不能仅仅停留在对作品字面的分析理解上, 而要抓住作品的“文眼”, 从 语言的变化、结构、用词、语气等方面体会作者的感情变化, 揣摩作者的言外之意, 弦外之 
音, 启发学生联想, 使他们对文章所描写的生活景象有如身临其境的感觉, 产生与作者情感 上的共鸣, 理性地把握作品的深刻寓意和思想内涵, 最终达到提高文学作品欣赏水平并在言 语实践中熟练运用语言的能力。

语文课本中的古诗如王安石的《泊船瓜洲》，历来为人们所传诵。诗中第三句“春风又绿 江南岸”的“绿”字, 是锤炼词语的范例。据说, 先后改掉了“到”、“过”、“入”等词, 最后才确 定“绿”。这个“绿”字为什么比“到”、“过”、“入”等词好？其原因是，“绿”本是表示色彩的形容 词, 用在这作动词最富表现力, 它生动而贴切地写出了生机蓬勃、春满江南的形象, 语言清 新而洗炼, 如果选用其它的词则达不到这样好的艺术效果。又如杜甫《送蔡希鲁都尉》诗中 的“身轻一乌过，枪急万人呼”两句，其中“过”这个动词具体而形象地刻画出蔡希鲁驰骋疆场 的矫捷雄姿, 它很难用“疾”、“起”、“下”等词替换。再看鲁迅的《孔乙己》，作者是这样介 绍主人公第一次出场的: “孔乙己是站着喝酒而穿长衫的唯一的人。”孤立地看, 我们看不出 它有何重要。可在小说里, 它是一个关键性的句子, 它点明了主人公的身份、地位以及性格 特点。“站着喝酒”的是贫困的短衣帮, 尽管孔乙己是一个满口“之乎者也”的“读书人”，似乎 与短衣帮截然不同, 而此时身陷贫困、潦倒的孔乙己也只能与短衣帮为伍, 无法挤入长衫客 的行列; 尽管这样, 他却又偏偏不肯脱掉那件长衫, 仍然要护着“读书人”的面子, 摆着“读书 人”高人一等的架子, 以此从精神上显示他较短衣帮要略高一筹。孔乙己这种拮据的经济状况 和迂腐的精神生活, 使二者之间存在着难以调和的矛盾。显而易见, 孔乙己之所以成为咸亨 酒店“唯一”的上不着天, 下不着地的这一特殊人物, 除其深层的社会根源外, 还与其存在的 自身矛盾也是分不开的。又如“孔乙己是这样的使人快活, 可是没有他, 别人也便这么过。” 这句话单独看也显示不出其重要性, 而置于小说的具体语境中则举足轻重, 它表明了孔乙己 既是人家的笑料, 又是社会的废物。其地位之低下, 暗示了他悲剧命运的必然性。鲁迅先生 以这样深沉的笔调, 对吃人的封建社会进行了深刻的揭露和抨击, 学生一经细细体味, 会在 心灵上产生强烈的震撼。如此一来, 学生在领悟文学作品深遂思想内容的同时, 还能学习语 言大师运用语言艺术的高超技巧。

一句话，文学作品的“艺术审美在人的精神生活中起着提升精神、完善人格、安顿灵魂、 终极关怀”的重要作用, ${ }^{[12]}$ 而语言学理论在语文教学中不仅有助于对“文学的第一要素”语言艺 术的分析, 也有助于学生文学艺术欣赏能力的提高, 进而达到学会语言、运用语言的理想境 界。

\section{5. 结束语}

上述问题, 仅是一孔之见, 略作不成文的诌议。任何一门科学都是博大精深的, 论述它 不可能以偏概全, 语言科学也不例外。在此, 我们仅以一斑之论, 显示其功效在语文教学中 的几个方面。随着语言科学的不断丰富和发展, 随着人们对语言科学认识和研究的不断深入, 我们深信, 语言学理论在人们的实践活动中, 会得到越来越广泛的应用, 尤其是在今后的语 文教改中，将更会起到其它任何学科难以替代的指导作用。

\section{致谢}

本文为中国江西省高校教改项目《高师语言学理论与基础语文教学实践问题研究》 （JXJG-13-2-4）的阶段性成果之一。

\section{References:}

[1] (Russian) Stalin. Marxism and linguistic problems. Beijing: People's Press, pp.38,1981.

[2] K.Lei and Q.Hong-wu. The restricted mechanism of semantic evolution: context and semantic schema. Journal of PLA University of Foreign Languages, Vol.40, pp.60-66, 2017. 
[3] Y.Fei-sheng and X. Tong-qiang. Linguistics outline. Beijing: Peking University Press, pp. 149-151, 2011.

[4] S.Shun-you. To return to context of semantic teaching . Language construction,Vol.0,pp. 4-7, 2017.

[5] Z.Zhi-gong. The modern chinese language. Beijing: People's Education Press,pp.255, 1982.

[6] W.De-chun and C.Chen. Modern rhetoric. Shanghai: Shanghai Foreign Language Education Press,pp.37-39, 1989.

[7] (Southern and Northern Dynasties)L.Xie, and F.Wen-lan Note. Wen xin diao long note. Beijing: People's Literature Publishing Press,pp.493, 2006.

[8] M.Ze-dong. A letter to Comrade Chen Yi on poetry. People 's Literature, Vol.22,pp.4-5,1978.

[9] (Russian) Gorky. On literature. Beijing: People 's Literature Publishing Press,pp.160, 1978.

[10] Chinese Department of Sichuan University. Chinese contemporary literature research materials: Cao Yu album. Chengdu: Sichuan University Press,pp.40-49, 1979.

[11] (Russian) Gorky. Gorky anthology (Vol. II). Beijing: People's Literature Publishing Press,pp.195, 1983.

[12] T.Shui-ping. Deepening the researches of aesthetics of literature and art and promoting the chinese aesthetic spirit. Journal of Jiangxi Normal University(Social Sciences), Vol.48,pp.11-21,2015. 